\title{
Propiedad, apropiación y robo en torno a la autoría
}

\author{
Andrés Mombrú ${ }^{1}$ \\ amombru56@yahoo.com.ar
}

\section{Resumen}

El tema de la autoría no solamente plantea temas éticos y legales, también afecta de modo muy determinante las prácticas de docencia e investigación. Este trabajo trata de analizar la relación entre los conceptos de autoría, de propiedad, de lo propio -que es parecido pero no es lo mismo-y de las diferentes formas de "robo", legal e ilegal que se perpetran en torno a la producción intelectual en el campo de las ciencias sociales y de las humanidades. Indaga sobre el modo en que ciertas tradiciones operan reproduciendo prácticas instituidas a nivel educativo y de la producción de conocimiento de modo asimétrico entre instituciones y docentes e investigadores, quienes suponen sustraerse de las relaciones de producción y las formas de dominio establecidas y sin embargo son atravesados por ellas.

Palabras clave: Autoría, derechos de autor, docencia e investigación

\begin{abstract}
The subject of authorship not only involves ethical and legal issues, it also affects the practices of teaching and researching in a very determinant way. This paper intends to analyze the relationship among the concepts of authorship, of ownership, of one's own -which is similar but is not the sameand of the different ways of "theft", legal and illegal, that are committed regarding intellectual production in the field of social sciences and humanities. It inquires into the way in which certain traditions operate reproducing instituted practices concerning education and knowledge production in an asymmetrical way among institutions, teachers and researchers, who believes to be subtracted from the relations of production and from the established forms of domination but, nevertheless, they are crossed by them.
\end{abstract}

Keywords: Autoría, derechos de autor, docencia e investigación

Una vieja canción del músico Moris, de la década del 60, Pato trabaja en una carnicería, decía en sus primeros versos: "Todo empezó con el chiste que decía, lo tuyo es mío y lo mío es mío, no comprendimos que eso sería, lo que algún día nos heriría." ¿Qué es fica. Docente e investigador en la Universidad de Buenos Aires y en la Universidad Nacional de Lanús. 
mío?, ¿qué es tuyo?, ¿qué es nuestro? El tema de lo propio y de la propiedad, es decir, de lo que es propio en un sentido personal y en un sentido colectivo, el de la apropiación indebida y el de la propiedad como un derecho, atraviesa el problema de la autoría. Lo propio y la propiedad parecen referir a cosas similares y sin embargo distan mucho de ser lo mismo. Lo que consideramos nuestro puede ser compartido o no y en diferentes términos. Podemos prestar un libro, sin que deje de ser nuestro, pero no podemos prestar algo que, siendo nuestro, no vaya a ser devuelto, por ejemplo un riñón. En este caso decimos que donamos o regalamos algo que nos pertenece, que nos es propio y que pasará a pertenecer, a ser propio de otro. Ese otro puede ser individual o colectivo. Un cepillo de dientes puede ser prestado, pero nadie sería calificado de egoísta porque no lo quiera compartir, se lo considera propio y privativo. Se hace entonces necesario distinguir la diferencia entre lo que es propio y lo que es propiedad.

Lo propio es personal, lo que pertenece a la propia persona porque hace a su posibilidad de ser. Nuestros órganos nos son propios y en un sentido extensivo se podría decir que nuestras ropas, nuestras herramientas, el lugar que habitamos, etcétera; es decir, todo aquello que se encuentra en función de nuestra existencia y es requerido para que sea garantizada de acuerdo a un principio de equidad. Al mismo tiempo, eso que es propio puede ser al mismo tiempo compartido colectivamente, como por ejemplo, compartir un techo, o una comida o una prenda. Por otro lado están aquellas cosas que nacen como propias de un colectivo: un país, un barrio, una calle, una plaza, una institución. Los espacios y las entidades públicas no tienen dueño; cualesquiera de ellas que no sean pasibles de libertad de tránsito, de uso, se torna privada. La defensa de lo propio personal implica un legítimo sentido de dignidad y en consecuencia, esa defensa, o ese reclamo, de respeto por lo propio siempre es justo. De igual modo sucede con lo propio colectivo, que puede pertenecer a una comunidad específica: un territorio, un patrimonio cultural, entidades físicas o morales. Cuando el valor que adquieren trasciende a la comunidad en la que tiene origen, puede convertirse en "bienes" de la humanidad. Y preferimos decir "bienes", ya que ese concepto tiene una acepción más laxa, más ambigua, en la que se incluye la connotación que también tiene el término "patrimonio", pero que no la reduce a una dimensión económica.

Aquellas entidades que son consideradas "patrimonio" de la humanidad, pierden la posibilidad de ser compradas o vendidas, pertenecen a la humanidad presente y futura; pero el lenguaje siempre deja traslucir la matriz ideológica de la que surge, y entonces caemos en la contradicción de llamar "patrimonio" que remite originariamente a lo que es propio del "pater" de raigambre netamente patriarcal y qué, al poder mensurarse económicamente, puede ser comprado o vendido, con algo que se coloca en un ámbito de excepción, precisamente porque se lo sustrae de la dimensión de lo mercantil. Más allá de estas contradicciones, el sentido de propiedad, difiere del de lo propio, personal o colectivo, ya que -paradójicamente- tiene un valor jurídico que no necesariamente está emparentado con la justicia. En otras palabras, las leyes pueden ser justas o estar orientadas a promover justicia, pero en sí misma no sólo no son garantía de justicia, sino que incluso pueden ser garantía de iniquidad. La dificultad para advertir esto es que en la sociedad capitalista, "justo" se ha tornado en un sinónimo de legal. Muchas veces la justicia no radica en el cumplimiento de la ley, sino en su incumplimiento. Pero si ese incumplimiento no va acompañado de una modificación en la correlación de fuerzas y 
Andrés Mombrú /Propiedad, apropiación y robo en torno a la autoría /47 en la instauración de otra escala de valores en lo que refiere al concepto y las prácticas de justicia, entonces las normas vigentes establecerán los criterios de justicia y se aplicarán de modo discrecional las penas. Los Estados, y muchos poderosos, incumplen con las leyes sin que esto genere -en buena cantidad de casos- castigo; pero esas mismas leyes se aplican de manera implacable a los más débiles. En nuestra sociedad hay incumplimientos que son considerados contravenciones y otros delitos. Ambos implicarían una violación de las leyes o normas establecidas, la diferencia radica en la pena. No entraremos aquí en tecnicismos jurídicos, pero hay acciones que son catalogadas como criminales, en ellas se encuentra en un lugar destacado los llamados "delitos" contra la propiedad.

Ser propietario, tener un título de propiedad, no es indicativo, per se, de que aquello que se encuentra registrado a nombre de un propietario le sea propio. La conquista del desierto otorgó títulos de "propiedad" de enormes extensiones de tierra a los conquistadores, legalmente son sus dueños y esa es su propiedad por derecho, sin embargo esos territorios son "propios" de aquellos que los habitaban y a los que les fueran arrebatados. He aquí una apropiación sujeta a derecho, pero realizada sobre una criminal injusticia. Injusticia porque viola lo que es justo, imponiendo por sobre ello lo que establecen las instituciones encargadas de impartir justicia como "derecho".

La autoría, por otra parte, tiene un sentido que muchas veces se torna ambiguo en lo que hace a "lo propio" y a la "propiedad". Preguntando una vez a un grupo de estudiantes de arte de quién era la obra "Los girasoles" -que en realidad es una serie de cuadros-, todos respondieron obviamente que de Vincent Van Gogh. Cuando les dije que se equivocaban, que "Los girasoles" no pertenecían a Van Gogh, todos quedaron desconcertados, como si esa declaración estuviera revelando alguna última novedad sobre el carácter apócrifo de la obra -cosa que ha ocurrido no pocas veces en el arte-. ¿Y, a quién pertenece?, preguntaron; en ese momento respondí que pertenecía al empresario japonés Yasuo Goto, quien la había comprado por cerca de 75 millones de dólares. Agregué que Yasuo era el propietario, pero que aquella obra pertenecía y pertenecería por siempre al pintor holandés. No sería tan triste la historia si Van Gogh no hubiera vivido y muerto en la más absoluta pobreza. A pesar de haber sido autor y de haberle sido propias sus obras como producto de su creatividad y maestría, los criterios de mercantilización de una sociedad en la que todo puede convertirse en mercancía, amparándose por lo tanto en un título de "propiedad", produjeron la "enajenación" de lo que era "propio". Es verdad también que el artista produce una obra que "dona" a la sociedad. El señor Yasúo Goto no podía hacer con ella otra cosa que la que puede hacer cualquier persona, aunque no haya pagado un centavo por ella, contemplarla. Luego las exhibiciones públicas o privadas pueden "socializar" la obra o seguir sacando dividendos como fruto de su apropiación.

Los conceptos de autoría y propiedad, presentan dificultades en su consideración, pues son asuntos que van por senderos diferentes. La suma de 75 millones de dólares pagada por el empresario japonés parece escandalosa, sin embargo, es una cifra insignificante en relación a los cientos y hasta miles de millones de dólares que grupos particulares obtienen mediante los derechos de patentes sobre producciones tales como desarrollos tecnológicos, medicamentos, producciones literarias, etcétera. Por ejemplo, producir medicinas cuyo enorme costo se desvanece cuando se la puede poner al alcance de millones de personas, 
producen, sin embargo, cientos de millones de dólares cuando se las comercializa por sumas enormes y para un mercado al que tienen acceso sólo sectores privilegiados de la sociedad. Menor costo mayor ganancia, es la lógica subyacente. La consecuencia es que la propiedad de las patentes, que las leyes y el Estado amparan, genera millones de personas sin acceso a ellas, que sufrirán y morirán. El criterio que gobierna todas estas producciones es el más alto y sagrado principio de nuestra sociedad, la "propiedad privada". La pregunta que nos hacemos aquí es, ¿̇de dónde han salido los insumos y los recursos materiales y humanos que permiten el conocimiento y la creatividad?

En general estos son bienes sociales, de producción colectiva, son el resultado del conocimiento acumulado por generaciones, del lenguaje del que nadie es dueño, de los recursos naturales sobre los que podemos servirnos con respeto por la naturaleza, o apropiarnos en base a "derecho" en provecho propio y en detrimento de la misma naturaleza y de la sociedad. Ahora bien, juntar todas esas cosas y convertirlas en algo de provecho tiene su mérito y merece su reconocimiento y su recompensa, pero, ¿cuál es el límite? ¿Cuál es el derecho a lucrar con algo al costo del sufrimiento, la destrucción y la muerte de muchos?

Entre "autoría" y "propiedad privada" se produce una tensión que no siempre es claramente visualizable. La autoría reclama sus "derechos", pero los únicos "derechos" que regulan la relación con las producciones es el "derecho de propiedad". El primero queda sometido al segundo. El autor puede "ceder", "vender", "donar" lo producido y esto cuando no ha trabajado desde el comienzo en condiciones en las que, eso producido, queda "enajenado" por las condiciones mismas de producción. Siempre hay una relación de asimetría entre quién "cede" y quien usufructuará los beneficios de la cesión. Cuando se trabaja para una institución, empresa u organismo que financia esa producción, el resultado pertenece al financista. Ahora bien, ese financista puede ser una entidad que puede socializarlo sin lucro, como los resultados de algunas investigaciones científicas que se producen por investigadores individuales o equipos de investigación, como sucede en algunas universidades, o en determinados y muy limitados centros de investigación. En otros casos, la principal meta del financista es el lucro y las ganancias que derivan de su comercialización, que no tienen como fin mejorar la vida de las personas, contribuir al interés colectivo, sino el lucro como un fin en sí mismo. En este sentido el autor puede ver al fruto de su autoría como una creación, como un bien que surge a través de él, pero que es el resultado de un proceso histórico y social, o puede pensar también que ésa es su propiedad y que tiene el derecho de lucrar con ella.

¿Hasta dónde se extiende el "derecho de autor" y de una "retribución económica" por el mismo? ¿En qué consisten esos "derechos”? En áreas como las artes -música, pintura, literatura, cine- los derechos de autor están medianamente regulados, aunque en relación a un contexto que en las últimas décadas ha cambiado enormemente y que no se ajusta a los tiempos actuales en el que las nuevas tecnologías han modificado sustancialmente el escenario.

En el campo de la investigación científica en ciencias naturales, los derechos de autor suelen estar "conculcados" por las instituciones financiadoras y por los sistemas de patentes. En el campo de la investigación en ciencias sociales la situación es similar en 
Andrés Mombrú /Propiedad, apropiación y robo en torno a la autoría /49 lo que refiere a investigaciones de campo y al lucrativo negocio de las consultoras. Pero en buena parte de las ciencias sociales y en las humanidades en general, la "autoría" es algo que no suele quitar el sueño en relación con los posibles beneficios económicos que se podrían obtener (insignificantes en general). Las entregas sistemáticas de la producción que surge de su seno suele ser a cambio -en el mejor de los casos- de algún tipo de "recompensa moral", de la posibilidad de obtener un prestigio o un reconocimiento que permita colocar al autor en otro orden de consideración, y/o le permita aspirar indirectamente a mejores oportunidades de trabajo, o premios y galardones si hay fortuna. Muchos no sólo son capaces de ceder "derechos", sino que estarían dispuestos a pagar para que sus producciones sean publicadas y sus nombres aparezcan junto a otros nombres prestigiosos. Aquí las tensiones no suelen ser, en general, por la obtención de recursos económicos; las disputas suelen darse más bien en el delicado juego de plagiarios y plagiados.

La apropiación indebida puede producir un beneficio que se obtiene con el esfuerzo o el talento de otro. Puede ser tan pequeño como el de los estudiantes que plagian trabajos de sus compañeros, o de otros autores, con el interés de aprobar un examen o una materia; incluso se puede llegar al punto de plagiar una tesis, ya sea por no tomarse la molestia de trabajar o por la falta de capacidad para hacerlo. La falta de rigor de muchos docentes en las correcciones hace que esos plagios circulen con mayor o menor impunidad y que esa clase de estudiantes hagan de la práctica su modus operandi durante los años de formación. Aunque sus consecuencias económicas inmediatas son prácticamente nulas, esta no deja de ser una situación grave, que viola la autoría, y de consecuencias nefastas al largo plazo, ya que una carrera cimentada en la mentira, el oportunismo y el plagio tiene como resultado a profesionales, académicos y científicos de pésima formación y de muy escaso conocimiento. En el mejor de los casos, estos "delincuentes" quedarán excluidos de las funciones a causa de su propia incapacidad. Si logran infiltrarse las consecuencias son más graves; de hecho, pueden producir "mala praxis" en cualquier disciplina que se trate. Pero el peligro más grande es cuando alcanzan cargos que implican responsabilidades y compromisos para los que no sólo están poco preparados, sino en los que el ejercicio de su función produce efectos de amplio impacto negativo y con consecuencias devastadoras en muchos casos.

Ahora bien, queremos referirnos exclusivamente a la producción intelectual que se expresa a través de la publicación -por medio de cualquier soporte disponible- de artículos y libros. Excluimos de estas consideraciones a la llamada "propiedad industrial", que refiere a las invenciones, marcas o patentes, comunes en los campos de la industria, y de desarrollos industriales a partir de investigaciones científicas. Entre las llamadas producciones que dan lugar al derecho de autor, también queremos dejar de lado en esta consideración a las producciones estéticas: pintura, escultura, o literarias, como novelas, cuentos, etcétera; o musical: partituras, letras, danza, etcétera. Nos referiremos solamente a libros, artículos y distintos "papers" como el producto final de la tarea de investigadores, sobre todo en el campo de las ciencias sociales. En algunos casos, los artículos científicos son sólo un modo de presentación de una autoría subyacente, por ejemplo: un desarrollo biomédico, algún tipo de dispositivo o incluso una teoría científica. En otros casos, vinculados a la producción intelectual -y quizá más próximos a la producción literaria-, el mismo artículo o libro es "la obra"; esto es, 


\section{0/ Perspectivas Metodológicas /19/Vol. II /Año 2017}

el objeto de autoría no radica en aquello a lo que la obra remite, (una vacuna), sino a la obra misma, el libro, el artículo, etcétera. En la República Argentina, el Registro Nacional de Propiedad Intelectual se realiza mediante una declaración con todos los datos que hacen a la datación de la obra y el depósito de ejemplares que deberán ir a parar a Biblioteca Nacional, Biblioteca del Congreso, Archivo General de la Nación y Dirección Nacional del Derecho de Autor.

El registro y el depósito de las obras a través de la Cámara Argentina del Libro parece estar destinado a proteger a los autores de abusos realizados sobre sus obras, que puede ser llevado adelante por editoriales o instituciones de investigación, y que se perpetra mediante el incumplimiento de las normas establecidas. El apartado "De las Penas", de la ley 11.723 de propiedad intelectual, en todos sus artículos refiere a las penalizaciones que se aplicarán a quienes incumplan lo dispuesto con penas de distinta duración y multas de diverso valor monetario. Pero lo establecido por la ley no logra impedir la situación de indefensión de los autores frente a los abusos, muchas veces difíciles de probar, que las propias editoriales o el negocio de la fotocopia infringen constantemente. Ahora bien, cuando se consultan los juicios llevados adelante en lo que refiere a propiedad intelectual, se puede advertir que en su mayoría pertenecen a reclamos sobre obras musicales o literarias, pero es casi inexistente la demanda sobre violaciones a las normativas referidas a la obra de intelectuales o científicos de las ciencias sociales. En los ámbitos universitarios, quienes suelen verse más perjudicadas son aquellas editoriales, en general pequeñas, que publican materiales de cátedra frente a fotocopiadoras externas o internas a las facultades que venden descaradamente materiales que le son ajenos. Es vergonzoso ver cómo, en la mayoría de las universidades, las fotocopiadoras autorizadas a funcionar en su interior violan con total impunidad todas las normas legales sobre autoría y sobre propiedad intelectual. En ocasiones los docentes-investigadores son víctimas cuando se publican sus producciones sin autorización, en otros casos son cómplices, -por ignorancia o por desconocimiento- del negocio ilícito de la fotocopia, cuando ellos mismos entregan a las fotocopiadoras materiales fotocopiados de libros que violan abiertamente los derechos de autoría y propiedad intelectual. Esta situación pone al descubierto instancias que revelan nuestras pobrezas intelectuales e institucionales en muchos aspectos.

Algunas gestiones universitarias han tenido una auténtica preocupación por facilitar el acceso a los estudiantes a obras de calidad y a costos muy accesibles. El ejemplo más trascendente corresponde a lo que fuera en otros tiempos la gestión de EUDEBA por Boris Spivacow entre 1958 y 1966, pero que dejará marcada su impronta por un tiempo más prolongado. EUDEBA no solamente publicaba los libros clásicos de todas las disciplinas que correspondieran a todas las carreras de la Universidad de Buenos Aires y también a autores de vanguardia, sino que además daba lugar, en su colección Manuales, a la publicación de muchos trabajos de docentes, investigadores, intelectuales, que desarrollaban sus actividades en esa Universidad. En aquellos tiempos, los modos de producción editorial eran mucho más costosos que hoy, y sin embargo se podía ver claramente que el lema "Libros para todos" se sostenía en ofrecer libros que todos podían comprar. Lo cual revela que no se trata de un problema de costos, sino de una decisión política. Aquellos años de esplendor fueron opacados por "La noche de los bastones largos" y por dictaduras y también por gobiernos "democráticos" que 
Andrés Mombrú /Propiedad, apropiación y robo en torno a la autoría /51 avasallaron a la educación pública, a la comunidad científica, a los intelectuales, muchos de los cuales fueron perseguidos y tuvieron que emigrar o pasaron a engrosar la lista de los asesinados y desaparecidos. La educación y la ciencia argentina, que habían alcanzado niveles de excelencia a escala mundial, tenían que ser desmanteladas, propiciando condiciones de insuficiencia que sólo fue remontada por la inquebrantable voluntad de los trabajadores de la educación.

La falta de financiamiento de políticas editoriales era parte del estado a que había sido sometida la educación superior, la que durante décadas ha contado con los recursos mínimos para sostener los gastos básicos y el sueldo a los docentes, amén de algunas gestiones que supieron usar muy discrecionalmente los recursos públicos llenando sus propios bolsillos. Por motivos que no viene a cuento analizar aquí, la educación primaria y secundaria no suele tener producciones propias, aunque ambas se convirtieron rápidamente en uno de los negocios editoriales más lucrativos de todos los tiempos a través de los monopolios de dos o tres editoriales que concentraban con un público cautivo todo el mercado. La multiplicidad y diversidad del mundo universitario hacía más difícil producir un manual para varios millones de estudiantes. Así y todo, siempre hubo editoriales que ofrecían textos a bajo costo. En ese clima, donde los mejores docentes e investigadores se encontraban en el exilio o expulsados de la universidad, y donde además los "libros baratos" no existían ni tampoco los apuntes de cátedra (mimeografiados) de autoría del profesor a cargo de la materia, se produce un reemplazo de autoridades y cuerpo docente de escasos dotes intelectuales. Los refritos y pastiches se convirtieron en el material de estudio de los alumnos universitarios. Estaba naciendo la cultura de la fotocopia, que se extendería hasta nuestros días y que se ha instalado de tal modo que no puede ser desplazada ni por los "libros baratos" ni por los soportes digitales. En los países "del primer mundo", que tanto se admiran por sus adelantos y avances en el campo de la educación, la titularidad de una cátedra no se alcanza si no se posee por lo menos una publicación propia del área disciplinar. Las mismas autoridades universitarias parecen desentenderse de este tema y, dentro de las propias instituciones, el negocio ilegal de las fotocopias se desarrolla con toda libertad por parte de concesiones y principalmente de los centros de estudiantes que, con la excusa de facilitar los materiales a los estudiantes, se valen de recursos como locales, energía, instalaciones, insumos, que pertenecen a la universidad y cuyo provecho se traduce, en ocasiones, o en parte, en ganancia propia y en financiamiento de sectores políticos de los que ganaron el centro. Ninguna de estas cosas es desconocida, no es ni siquiera "un secreto a voces", es algo a la vista de todos. Esta dinámica se ha convertido en el modus operandi ya internalizado y aceptado en las comunidades universitarias. La lucha por los centros es, al mismo tiempo, la lucha por la caja, en la que se encuentran involucrados miembros de la comunidad académica.

El doble carácter de docente-investigador impone la producción y publicación de las investigaciones y su acceso a la práctica docente. En 1993, a través de la Secretaria de Políticas Universitarias, se implemente el sistema de incentivos para docentes investigadores.

Si bien el objetivo declarado fue aumentar la investigación y desarrollar una carrera académica integrada -docencia junto a investigación-, las características del Programa (plus salarial, escalafón jerárquico, sistema de 


\section{2/ Perspectivas Metodológicas /19/Vol. II /Año 2017}

evaluación) muestran que el verdadero sentido fue transformar las relaciones entre el Estado y las universidades a través de una injerencia directa en las actividades que debía realizar un docente universitario. Mediante la introducción del Programa se buscó que los docentes realizaran más investigación científica, pero también se intentó tener un mayor control por parte del Estado a través de la SPU, de lo sucedido en las universidades en materia de investigación y respecto al conjunto de las actividades de los investigadores universitarios. En los considerandos del decreto de creación del Programa se le asignó, asimismo, un rol destacado a las universidades en la producción de conocimiento. El Programa declaró como objetivo: "promover el desarrollo integrado de la carrera académica en las Universidades Nacionales", entendiendo por ello la realización conjunta de actividades de "docencia, investigación, extensión y gestión", como respuesta a una serie de circunstancias:

- La baja proporción de docentes universitarios que participan en actividades de investigación científica y tecnológica (15\% del total de docentes).

- La importancia del desarrollo científico y tecnológico para el crecimiento económico y la justicia social, en el marco de un nuevo paradigma científico-tecnológico dominante a escala mundial, basado en la microelectrónica, la biotecnología y los nuevos materiales, que ubica a las Universidades como destacados protagonistas en la investigación científica y en el desarrollo de nuevas tecnologías de productos y procesos.

- La necesidad de aumentar las tareas de investigación y desarrollo a nivel nacional y de fomentar la reconversión de la planta docente, motivando una mayor dedicación a la actividad universitaria y a la creación de grupos de investigación.

- La simultánea contribución del Programa al mejoramiento de los ingresos de los docentes universitarios durante el período en que participen en proyectos de investigación, en el marco de asignación de recursos a las Universidades en función de programas específicos basados en criterios objetivos que favorezcan el rendimiento del trabajo académico. (Sarthou, Araya, 2015).

La creación de una Secretaría de Políticas Universitarias hubiera supuesto un contrasentido en otros períodos históricos, donde la competencia del Estado se limitaba al financiamiento y el funcionamiento de las mismas estaba basado en el gobierno autónomo. Dotar a una Secretaría con poder de intervención suponía el primer paso hacia la mayor regulación y orientación de las actividades docentes y de investigación de las universidades. (Gordon, 2011, p. 173, en Sarthou, Araya, 2015)

Más allá de las delicadas situaciones que plantean los autores citados, la situación de los docentes se ve modificada en sus prácticas cotidianas. Los docentes nunca tuvieron buenas remuneraciones y su papel, en general, se reducía a ser trasmisores de conocimientos, pero no a ser productores de los mismos. Aquí se produce una nueva tensión que dejaremos de lado pero que no conviene perder de vista; la mayoría de los docentes universitarios carecen de formación pedagógica, ya que las universidades han privilegia- 
Andrés Mombrú /Propiedad, apropiación y robo en torno a la autoría /53 do durante décadas a sus propios egresados con perfil académico o profesional pero no instrucción para la docencia, la que sí poseen los docentes formados en los profesorados, aunque careciendo de la formación necesaria para la investigación.

La nueva figura que aparece del docente-investigador, si no renguea de una pata renguea de la otra. Ahora bien, de pronto, la mayoría de los docentes que no ven la oportunidad de mejorar sustancialmente sus ingresos mediante los incentivos económicos personales, pero sí de la necesidad de participar de la carrera docente con nuevas reglas, se encuentran, sin embargo, muy poco preparados para encarar eso que se denomina "investigación". Sólo las camadas de docentes más jóvenes, que comenzaron sus actividades con los programas y las convocatorias ya en funcionamientos, pudieron encajar mejor en el nuevo sistema. Pero este nuevo rol de docente-investigador implicaba la suma de nuevas tareas; a las ya agobiantes de la docencia en universidades de ingreso irrestricto, o más abiertas a la comunidad, con las tareas habituales de planificación y preparación de clases y contenidos frente a cursos muy numerosos, a cientos de exámenes que corregir y a las nuevas necesidades de preparación y de aplicación de otros fenómenos que imponía también nuevas exigencias, el uso de las Tecnologías de la Información y la Comunicación y sus aplicaciones educativas, se sumaban las ignotas actividades de investigación. Ante esto no había muchas opciones, más que concentrar esfuerzos en todos esos frentes unificando los temas docentes con los de investigación y aplicación.

¿De qué modo inciden todos estos elementos en las prácticas de la docencia y la investigación, y en la exteriorización y divulgación de la producción académica e intelectual? ¿En qué medida lo que el esfuerzo -y los recursos- de los proyectos de investigación y la formación de nuestros docentes e investigadores producen, repercute en las condiciones de realización de la docencia? ¿Cómo las instituciones reconocen y estimulan esas producciones, protegiendo el trabajo intelectual que dinamiza la práctica docente y que va mucho más allá de cumplir un horario, dar un programa y atender a todas las instancias burocráticas? Cuando se observan programas y bibliografías que han permanecido inalteradas durante más de 20 y a veces 30 años, se puede concluir que, en no pocos casos, la investigación no ha aportado nada a la docencia, pues ella se ha convertido en una tarea enajenada donde el único interés del docente es cobrar su salario y la del investigador continuar manteniendo esa categoría. En el caso de muchos investigadores, esa situación se hace patente cuando su única actividad es solicitar que se los incluya como "investigador" dentro de un proyecto y comunicar si mandó alguna ponencia a un congreso o publicó algún artículo -que muchas veces no tiene ninguna relación con la investigación- para incluir en los informes de avances o en el informe final.

Esta situación generó dos categorías de docentes: los docentes y los docentes-investigadores. Los primeros parecen quedarse a la saga de promociones mediante concursos e incentivos económicos, miran con desdén a sus compañeros investigadores contando los días para la jubilación. Los segundos, con aparentemente más opciones, también pueden dividirse en dos: los que hacen como sí y los que corren desesperados detrás de plazos, vencimientos, solicitudes perentorias; los que le quitan horas al sueño para entregar el artículo, el "paper", el informe, la solicitud de convocatoria en los malditos sistemas que se caen todo el tiempo. Ninguno de ellos parece muy afortunado. 


\section{4/ Perspectivas Metodológicas /19/Vol. II /Año 2017}

Se podría decir que la publicación de libros y artículos, la participación en congresos, el desarrollo de la carrera docente y de investigador, tiene una recompensa moral, al sentir que se cumple con la tarea, y una recompensa institucional a la hora del puntaje que se puede obtener en un concurso, o cuando se pretende una beca; pero si los contenidos son "ficcionales" y la calidad de la producción es mediocre, entonces los puntos pueden llegar, pero la calidad como docente y como investigador se ve severamente desmerecida. $\mathrm{Si}$ a esto se le suma que los incentivos por investigación son míseros, que en muchas universidades los concursos son escasos, que muchos docentes se encuentran durante años, y a veces durante décadas, en carácter de contratados o interinos, con cargas horarias abrumadoras y demandas burocráticas absurdas - por ejemplo, mantener al día tres o cuatro sistemas de información curricular- entonces el problema no radica solamente en los aspectos legales que hacen a la autoría, sino en los contextos materiales y en las condiciones de posibilidad de la docencia, la investigación y la extensión; principalmente esta última, que es la que permite la transferencia de producciones y de conocimientos al conjunto de la sociedad y que para un docente-investigador, sobre todo en el área de las ciencias sociales, se expresa como publicaciones.

Ahora bien, cuando los esfuerzos legítimos de la actividad de docencia e investigación se traducen en publicaciones que tienen alcance en las áreas de la docencia donde hay un público cautivo -el conjunto de los estudiantes que no eligen comprar un material, sino que no tienen más remedio- entonces la asimetría entre las editoriales grandes y los autores, entre las editoriales pequeñas y las fotocopiadoras, entre los comerciantes y el público, reproduce la enajenación del trabajo intelectual, que deja de estar incentivado como una producción social de conocimiento y se convierte en el lucro de unos pocos.

¡Cómo se equivocan los que piensan que las condiciones del trabajo intelectual se encuentran por encima, o sustraídas, de las vicisitudes de expoliación y robo que se ciernen sobre el trabajo manual!

\section{Bibliografía}

- Sarthou, N. y Araya, J. M. (2015). "El Programa de Incentivos a Docentes Investigadores en Argentina: a dos décadas de su implementación”. Ciencia docencia y tecnología, 26(50), pp. 1-34. 